\title{
Control of two-phase flow in a microfluidic system using ac electric fields
}

\author{
H. Morgan ${ }^{\mathrm{a})}$ and N. G. Green \\ School of Electronics and Computer Science, University of Southampton, Highfield, Southampton SO17 1BJ, \\ United Kingdom

\begin{abstract}
A. Ramos and P. Garcia-Sanchez
Department Electronica y Electromagnetismo, Universidad de Sevilla, Avda Reina Mercedes s/n, Sevilla 41012, Spain
\end{abstract}

(Received 2 October 2007; accepted 23 November 2007; published online 18 December 2007)

\begin{abstract}
The control and handling of fluids are central to many applications of the lab on chip. We report how alternating current (ac) electric fields can deflect and manipulate coflowing streams of two different electrolytes within a microfluidic channel. The two different electrolytes flow side by side over an array of interdigitated electrodes which occupies the width of the channel. Application of a $20 \mathrm{~V}$ (peak to peak) voltage at $1 \mathrm{MHz}$ to the electrodes causes the liquid with higher conductivity to occupy a larger region of the channel. This effect causes a significant displacement of the boundary between the two fluids. (C) 2007 American Institute of Physics. [DOI: 10.1063/1.2825835]
\end{abstract}

Dynamic control of fluids is important in lab on chip (LoC) technology, and microelectromechanical system devices are often used for this. ${ }^{1}$ However, such devices are complicated to construct and cannot be easily integrated into the LoC. Solid-state control of liquids using dc electric fields has also been widely used to manipulate fluids, particularly with capillary electro-osmosis. However, the high voltages required produce electrolysis and the electrodes must be placed outside the device to reduce the effects of Joule heating and gas bubbles. Electro-osmosis has been used in the LoC to enhance mixing of fluids, ${ }^{2,3}$ reduce diffusion (sample stacking), ${ }^{4}$ switch fluid streams, ${ }^{5}$ and focus flows. ${ }^{6}$

An ac electric field-induced fluid flow can also be used to move fluids and analytes or produce mixing. ${ }^{7-10}$ ac-based systems have many advantages, including lower power requirements, simple integration, and little or no electrolysis issues. Fluid is driven and controlled using microelectrodes that are fabricated within microchannels. The electrohydrodynamic forces produced by the ac fields act either on the bulk fluid (e.g., electrothermal flow ${ }^{7}$ ), or the double layer at the solid-electrolyte interface, ac electro-osmosis. ${ }^{7}$ In this letter, we describe a method for controlling the behavior of two coflowing streams of electrolytes in a microfluidic system using ac electric fields generated by a microelectrode array.

The experimental arrangement consists of a microdevice with microelectrodes fabricated on a planar glass substrateFig. 1. The electrodes are made from Pt and are $200 \mathrm{~nm}$ thick, manufactured using ion beam milling of the metal. They have a width and gap of $20 \mu \mathrm{m}$. The microfluidic channel width is $1 \mathrm{~mm}$ and has a height of $100 \mu \mathrm{m}$ and is fabricated from a photosensitive laminate resist. The channel is closed with an upper glass layer and the finished device clamped to seal the system. The electrodes have a chevron pattern (Fig. 1). An ac potential difference is applied to each alternate electrode, producing an electric field at the boundary of the two fluids along the length channel. The ac signals $\left(<20 \mathrm{~V}_{\mathrm{pp}}\right)$ were applied using signal generators with frequencies high enough to avoid electrode polarization and electrolysis.

\footnotetext{
${ }^{a)}$ Electronic mail: hm@ecs.soton.ac.uk.
}

Two different electrolytes are pumped across the electrodes, as shown in Fig. 1. Solutions of $\mathrm{KCl}$ with different conductivities were used, one ten times higher than the other. The $\mathrm{KCl}$ with the higher conductivity also contained a dilute solution of carboxyfluorescein, which enabled discrimination of the two separate flows and the boundary between them. When a single phase ac potential was applied to the electrodes, it was observed that the boundary between the two fluids moved, as shown schematically in Fig. 1. Two images from the device showing this effect are reproduced in Fig. 2. For a video of this, refer to Ref. 11. These data were obtained with an applied signal of $20 \mathrm{~V}$ (peak to peak) at a frequency of $1 \mathrm{MHz}$, with electrolyte conductivity of $\sigma_{1}=15 \mathrm{mS} \mathrm{m}^{-1}$ and $\sigma_{2}=1.5 \mathrm{mS} \mathrm{m}^{-1}$.

When the voltage was applied, the boundary between the two fluids moved to the region of lower conductivity, within a few tens of milliseconds after applying the potential. This boundary then remained fixed along the entire length of the channel downstream, even after the electrode array had ended (Fig. 1). When the voltage was switched off, the fluid returned to the steady-state position. This effect was observed for frequencies around $1 \mathrm{MHz}$, but when the frequency of the applied signal was increased to $10 \mathrm{MHz}$, the effect was no longer seen. Changing the electrolyte conductivities to $\sigma_{1}=15 \mathrm{mS} \mathrm{m}^{-1}$ and $\sigma_{2}=20 \mathrm{mS} \mathrm{m}^{-1}$ greatly reduced this effect, but a small deviation in the boundary was still seen. Changing the voltage altered the position of the boundary. At voltages of less than $5 \mathrm{~V}$, little effect was observed. As shown in Fig. 2, with $20 \mathrm{~V}$ applied the high con-
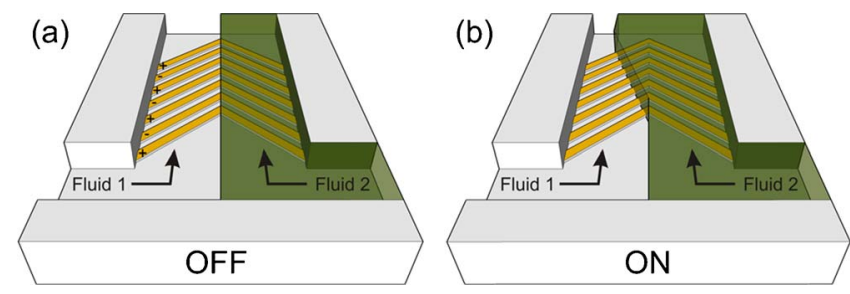

FIG. 1. (Color online) Diagram of the experimental chip showing the two coflowing fluid streams before and after application of the electric field. The electrode array is on the bottom of the channel and each alternate electrode is connected to a single phase voltage supply. 


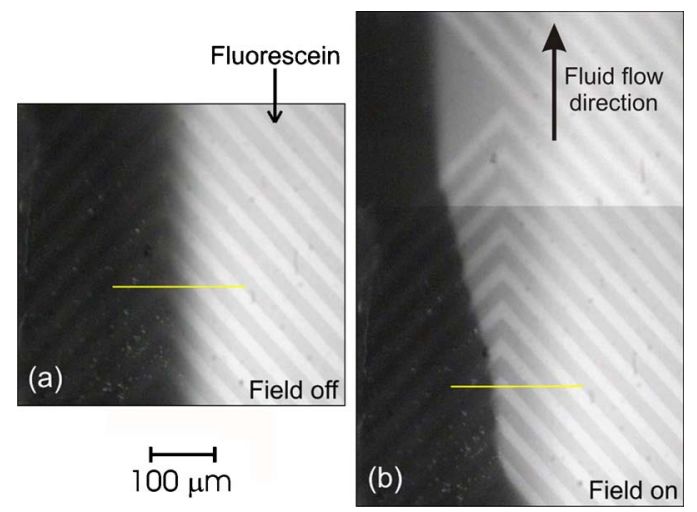

FIG. 2. (Color online) Experimental images of the two electrolyte streams: (a) before and (b) after application of $20 \mathrm{~V}$ peak to peak at $1 \mathrm{MHz}$. Note that the deflection continues downstream after the electrode array finishes. For a video of this effect see Ref. 11.

ductivity liquid almost occupied the entire width of the channel. This data were obtained with a volume flow rate of $0.3 \mu \mathrm{l} \mathrm{s}^{-1}$, equivalent to an average fluid velocity of $2 \mathrm{~mm} \mathrm{~s}^{-1}$.

The electrical body forces on a liquid are described by the following equation: ${ }^{12}$

$$
\mathbf{f}_{e}=\rho_{q} \mathbf{E}-\frac{1}{2} E^{2} \nabla \varepsilon+\frac{1}{2} \nabla\left[\rho_{m}\left(\frac{\partial \varepsilon}{\partial \rho_{m}}\right) E_{T}^{2}\right],
$$

where $\rho_{q}$ is the charge density, $\rho_{m}$ the mass density, $\varepsilon$ the permittivity, $T$ the temperature, and $E$ the magnitude of the electric field. The first and second terms are the Coulomb and dielectric forces, respectively, and the last term is the electrostriction force, which can be ignored for incompressible fluids. ${ }^{12}$ For the system described here, the dielectric term is zero because both liquids have the same permittivity. Electrolytes are overall neutral (no excess free charge); and the Coulomb force acts on the free charge at the interface between the liquids. Therefore, the electric field at the boundary creates a force which displaces one liquid with respect to the other. The free charge is induced by the electric field, which is also responsible for the force. Therefore, the time average force is nonzero and scales with $E^{2}$.

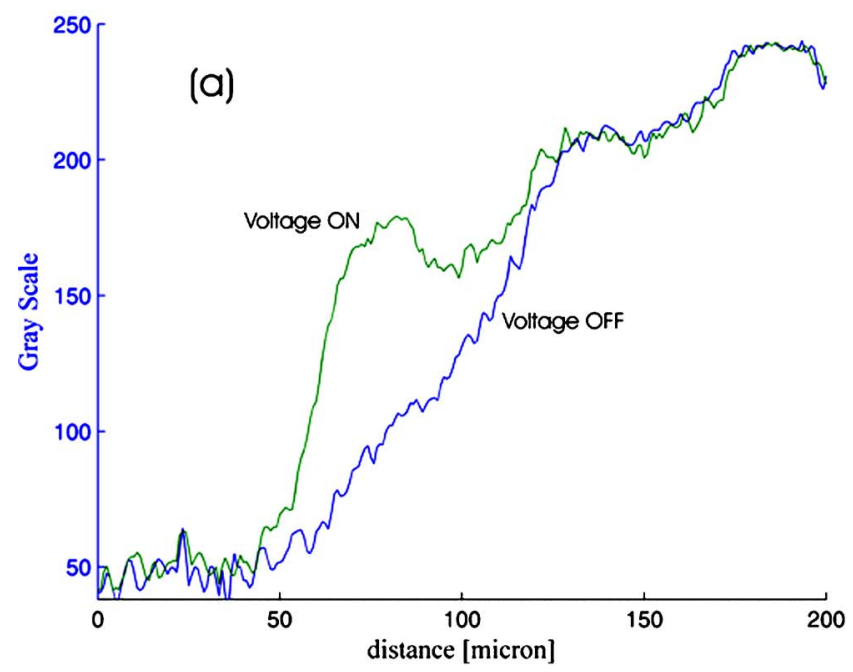

The behavior of the liquid is in many ways analogous to the behavior of a polarizable particle in a nonuniform electric field - a phenomena known as dielectrophoresis (DEP). ${ }^{13,14}$ At low frequencies, the free charge dominates, but as the frequency increases, the permittivity dominates because there is no time for charge to accumulate at the interface. Therefore, as in DEP, permittivity effects are predominant at high frequencies, and for two fluids of identical permittiviting, the force is zero. At low frequencies, free charge dominates and the liquid with higher polarizability (the higher conductivity) tries to maximize the electrical energy of the system (at constant potential) and occupies the region of high electric field, as observed in these experiments.

For the two fluids used in this work, the relaxation frequency of the interfacial free charge is ${ }^{13}$

$$
f_{2}=\frac{1}{2 \pi} \frac{d_{1} \sigma_{2}+d_{2} \sigma_{1}}{d_{1} \varepsilon_{2}+d_{2} \varepsilon_{1}}
$$

where $d$ is the thickness of the dielectric. In this case, $d_{1}$ $=d_{2}$ and $\varepsilon_{1}=\varepsilon_{2}$ so that $f_{2}=1.85 \mathrm{MHz}$. Therefore, for frequencies much greater than $f_{2}$, the force is negligible, but for frequencies much lower than $f_{2}$, the force is constant. Our experiments concur with this simple estimation. The magnitude of this force scales with the difference in conductivity of the two liquids, according to $\left(\sigma_{2}-\sigma_{1}\right) /\left(\sigma_{2}+\sigma_{1}\right)$. For the two conductivities shown in Fig. 2, the factor is 0.82 , but for the two samples of nearly equal conductivity, the factor is only 0.14 , i.e., the force is much reduced. The two fluids were driven by gravity. Changing the height of the fluid reservoirs by the order of $1 \mathrm{~mm}$ corresponds to a hydrostatic pressure of the order of $10 \mathrm{~Pa}$. This pressure change was enough to produce an observable change in the position of the interface. For a voltage of $20 \mathrm{~V}_{\mathrm{pp}}$, the time-average electrical pressure in the system can be estimated to be approximately $40 \mathrm{~Pa}$, which is more than enough to cause the deflection observed.

Interestingly, Fig. 2 also shows that, when the field is applied, the boundary between the two fluids is compressed and better defined. Upstream of the electrodes, the boundary is broad due to diffusion of the fluorescent dye into the liquid, but over the electrodes, the boundary becomes narrower. Figure 3(a) shows a profile of the light intensity (propor-

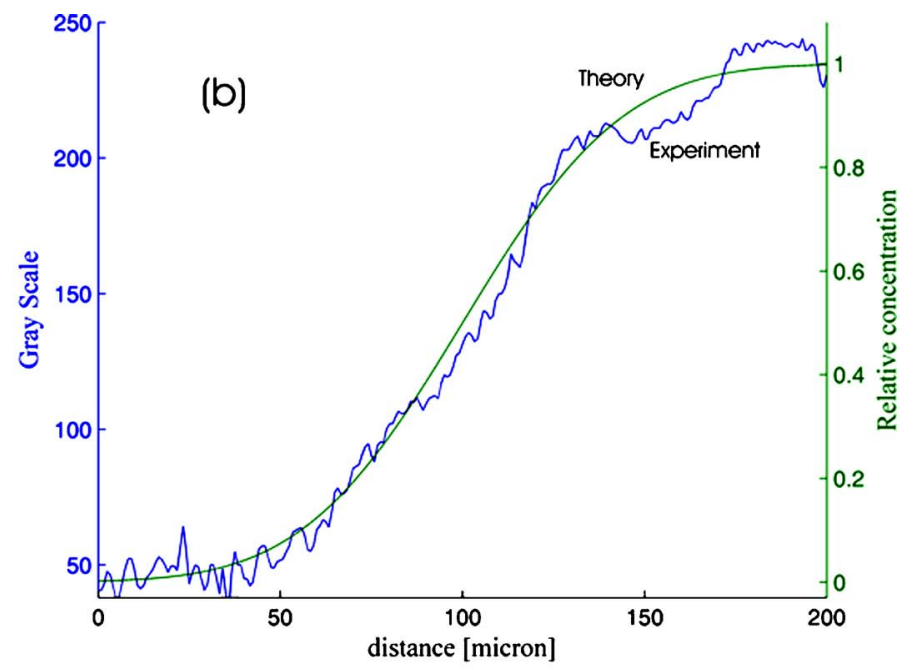

FIG. 3. (Color online) Profiles of the light intensity across the interface: (a) with and without electric field; (b) comparison of the experimental concentration profile and the theoretical profile (without field).

Downloaded 01 Feb 2008 to 152.78.66.11. Redistribution subject to AIP license or copyright; see http://apl.aip.org/apl/copyright.jsp 
tional to concentration of fluorescein) across the interface before and after application of the voltage, determined along the lines (200 $\mu \mathrm{m}$ long) shown in Fig. 2. Figure 3(b) shows that the intensity profile (with the voltage off) is well matched by the theoretical (normalized) concentration (of fluorescein) calculated from the diffusion equation. The observation point was $3.1 \mathrm{~mm}$ from the junction of the two fluids (diffusion time of $1.5 \mathrm{~s}$ ), and the curve was calculated using a diffusion constant for fluorescein of 4 $\times 10^{-10} \mathrm{~m}^{2} \mathrm{~s}^{-1}$. ${ }^{15}$ When the voltage is applied the profile across the boundary changes markedly, as shown in Fig. 3(a), appearing to reduce the effect of diffusion.

Although dc fields have been used to limit the diffusion of charged species, ${ }^{4}$ this has not been previously observed with ac fields. The origin of this effect is not clear at present, but may be related to the polarization of the interface.

In conclusion, we have demonstrated that applying an ac electric field across the boundary of two electrolytes with different conductivities in a microchannel generates a force at the interface that alters the position of the boundary. We have shown qualitative agreement between experiment and theory in terms of frequency and voltage dependence. Further work is required to quantitatively characterize these phenomena. The ability to actively deflect fluids within a microsystem has many technological applications, for example, active solid-state deflection of streams into different outlets for sorting or analyzing of particle and analytes. The phenomenon could also be used to rapidly switch different fluid stream to different sensing points, allowing multiple analysis steps to be performed in a single microfluidic channel. Dy- namic control of flow focusing of fluids should be possible, in a manner analogous to DEP-focusing of particles along the center of a channel. ${ }^{16}$ With a high voltage $\left(\sim 40 \mathrm{~V}_{\mathrm{pp}}\right)$, electrohydrodynamic instabilities were produced at the boundary which could be exploited to drive chaotic mixing, as demonstrated for dc fields. ${ }^{2}$

${ }^{1}$ M. Koch, A. Evans, and A. Brunnschewiler, Microfluidic Technology and Applications (Research Studies Ltd., Baldock, Herts, England, 2000).

${ }^{2}$ M. H. Oddy, J. G. Santiago, and J. C. Mikkelsen, Anal. Chem. 73, 5822 (2001).

${ }^{3}$ I. Glasgow, J. Batton, and N. Aubury, Lab Chip 4, 558 (2004).

${ }^{4}$ S. C. Jacobson and J. M. Ramsey, Electrophoresis 16, 481 (1995).

${ }^{5}$ A. Y. Fu, C. Spence, A. Scherer, F. H. Arnold, and S. R. Quake, Nat. Biotechnol. 17, 1109 (1999).

${ }^{6}$ S. C. Jacobson and J. M. Ramsey, Anal. Chem. 16, 3212 (1997).

${ }^{7}$ A. Ramos, H. Morgan, N. G. Green, and A. Castellanos, J. Phys. D 31, 2338 (1998).

${ }^{8}$ M. Z. Bazant and T. M. Squires, Phys. Rev. Lett. 92, 066101 (2004).

${ }^{9}$ N. Sasaki, T. Kitamori, and H. B. Kim, Lab Chip 6, 550 (2006).

${ }^{10}$ H. Zhao and H. H. Bau, Phys. Rev. E 75, 066217 (2007).

${ }^{11}$ See EPAPS Document No. E-APPLAB-91-020752 for a video showing the movement of fluid when the field is applied. This document can be reached through a direct link in the online article's HTML reference section or via the EPAPS homepage (http://www.aip.org/pubservs/ epaps.html).

${ }^{12}$ J. A. Stratton, Electromagnetic Theory (Mcgraw-Hill, New York, 1941).

${ }^{13}$ H. A. Pohl, Dielectrophoresis (Cambridge University Press, Cambridge, 1978).

${ }^{14}$ H. Morgan and N. G. Green, AC Electrokinetics: Colloids and Nanoparticles (Research Studies, Baldock, UK, 2003).

${ }^{15}$ C. T. Culbertson, S. C. Jacobson, and J. M. Ramsey, Talanta 56, 365 (2002).

${ }^{16}$ H. Morgan, D. Holmes, and N. G. Green, IEE Proc.: Nanobiotechnol. 150, 76 (2003). 